\title{
An Animal Model for the Study of Lung Protective Therapies During One Lung Ventilation (OLV) in Children
}

\author{
Thomas L. Miller, Andrew T. Costarino, Alicia Olivant, Doyle Lim, Thomas H. Shaffer and \\ Mary C. Theroux*
}

AI duPont Hosp for Children, Department of Anesthesiology and Critical Care \& Nemours Biomedical Research, and Thomas Jefferson University, USA

\begin{abstract}
One-lung ventilation (OLV) is the intentional collapse of one lung during surgical procedures in the chest. The objective of this report is to demonstrate that a piglet model of pediatric OLV mimics the clinical sequelae, and thus is valid to study lung protective interventions. Pediatric pigs $(\mathrm{n}=7 ; 21 \pm 1 \mathrm{~d}$ old; $7.7 \pm 0.5 \mathrm{~kg})$ were instrumented and exposed to OLV (left lung collapsed) for $3 \mathrm{hrs,} \mathrm{followed} \mathrm{by} 30 \mathrm{~min}$ of bilateral lung ventilation (BLV) before sacrifice and tissue harvest. Pulmonary compliance $(\mathrm{Cp})$ and resistance $(\mathrm{Rp})$ were measured half-hourly and plasma samples were drawn hourly for inflammatory cytokine analysis. Cp dropped to $86 \%$ and Rp rose to $148 \%$ of baseline with OLV, which is consistent with clinical observations given the anatomical differences of the pig thorax. Plasma levels of TNF- $\alpha$ and IL6 rose throughout the protocol, consistent with clinical observations. Lung histology showed greater atelectasis in the collapsed lungs following $30 \mathrm{~min}$ of BLV. The piglet model is valid for the study of OLV-induced lung injury and may be useful for the study of potential therapies to improve outcomes in the pediatric population.
\end{abstract}

Keywords: Thoracoscopic surgery, inflammation, lung injury, pulmonary mechanics, animal model, one lung ventilation.

\section{INTRODUCTION}

One lung ventilation (OLV) is the intentional collapse of one lung during surgical procedures in the chest, such as surgery on the thoracic spine, the esophagus or even on the lung itself [1-3]. Thoracoscopic surgical procedures require that a lung be collapsed to enable the surgeon to visualize and have safe access to the surgical field; thus, in order to facilitate performance of the surgical procedure, an injurious procedure needs to take place on an otherwise normal organ, namely the lungs. Subsequent to advances in technology, there has been a surge in these procedures since 1995. At our center alone (Alfred I. duPont Hospital for Children, Wilmington, DE), approximately 40-60 OLV dependent surgeries are performed each year.

Duration of the OLV is dependent on the surgical procedure and is typically between 2 to $8 \mathrm{hrs}$. The injury incurred by OLV, both pulmonary and systemic, is difficult to quantify clinically because of the concurrent trauma from the primary surgery and invasive nature of certain measurements. Clinical outcomes in these patients vary from full recovery to needing supplemental oxygen for several days, with tachypnea and atelectasis. The alternative to OLV dependent thoracoscopic surgeries is an open thoracotomy approach, which results in a greater degree of post-operative pain, a larger surgical scar and greater patient dissatisfaction. Therefore, research is needed to characterize the lung injury induced by OLV and refine the technique.

*Address correspondence to this author at the Nemours Biomedical Research, Alfred I. duPont Hospital for Children, Delaware 19803, USA; Tel: 302-651-5321; E-mail: mtheroux@nemours.org
The aim of this paper is to introduce an in vivo piglet model of OLV that parallels the clinical scenario, but allows for routine serum analysis and tissue harvest. Whereas a number of clinical studies have addressed lung functional parameters and demonstrated inflammatory injury related to OLV $[4,5]$, these studies are limited in that the clinical scenario offers poor experimental control and lung samples are typically unavailable due to the obvious risk to the patient. In addition, these clinical studies, largely performed in adults, are fraught with unavoidable issues such as the patient's underlying systemic illness, which is often a malignancy located in the thoracic region. There are a number of confounding factors that can potentially influence the appropriateness of an animal model to mimic the outcomes of human OLV. This report is intended to demonstrate the adequacy of a piglet model, as well as qualify the relevant differences in piglet versus human anatomy and respiratory mechanics, in order to support a line of studies aimed at lung protection during OLV. Our hypothesis was that we could develop an animal model of 'clinical OLV' by using a bronchial blocker with oral intubation, and that this model would demonstrate the same clinical features as patients subjected to OLV. More importantly, this model will serve as the platform through which we investigate the contribution of OLV related injury to surgical outcomes and explore therapeutic options.

\section{MATERIALS AND METHODS}

\section{Experimental Model}

Juvenile piglets were pre-sedated, orally intubated and then maintained on an anesthesia ventilator (Ohmeda 7000 ventilator, Ohmeda, Madison, WI; Modulus anesthesia system, Ohio Medical Products, Madison, WI) with the $\mathrm{FiO}_{2}$ held at 1 . After a 30 minute stabilization period, the left main 
stem bronchus was blocked using an Arndt pediatric endobronchial blocker set (Cook Critical Care, Bloomington, IN) and the animal was repositioned such that the ventilated (right) lung was gravitationally dependent. The left lung was preferred for blocking as the porcine right lung has an additional bronchus (apical bronchus) in addition to the right main stem bronchus, rendering the blocking of right lung technically difficult. Fig. (1) shows the two primary bronchi associated with the right lung. A $5 \mathrm{~mm}$ trocar (Ethicon-Endosurgery, Somerville, NJ) was introduced into the left thoracic cavity to simulate introduction of a surgical thoracoscope. For purposes of developing the model, the duration of OLV used for this report was 3 hours, based on average current clinical standards. Bilateral ventilation (BLV) was reestablished for endpoint measurements.

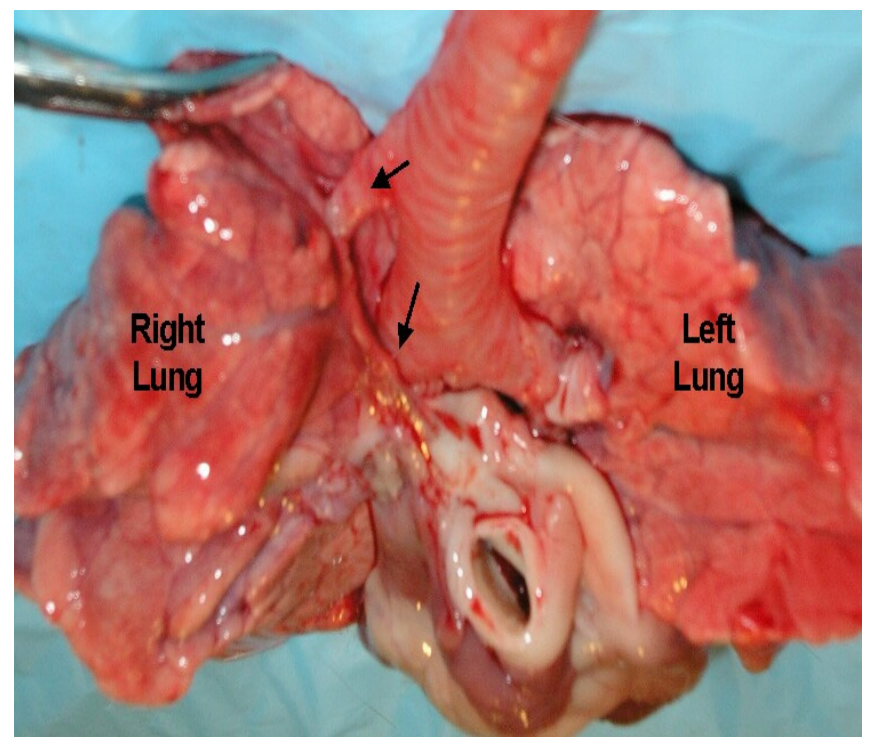

Fig. (1). Image of pig lungs demonstrating there are two right side primary bronchi (arrows). For this reason, the one lung ventilation (OLV) model was achieved by blocking the left lung.

\section{Instrumentation Procedure}

All animal procedures used for the establishment of this protocol were approved by the Life Science Center Animal Care and Use Committee, Department of Biomedical Research, Nemours, and were in accordance with National Institutes of Health guidelines. Piglets approximately 3 weeks old were anesthetized by injections $(2 \times 1 \mathrm{ml} / \mathrm{kg})$ of an anesthesia mixture (ketamine: $23 \mathrm{mg} / \mathrm{ml}$; azepromazine: 0.1 $\mathrm{mg} / \mathrm{ml}$; and xylazine: $0.05 \mathrm{mg} / \mathrm{ml}$ [KAX]) adapted from previously described protocols $[6,7]$. Prior to instrumentation, animals were placed on a radiant warmer bed (Resuscitaire ${ }^{\mathbb{B}}$; Hill-Rom Air-Shields, Hatboro, PA) to maintain a rectal temperature of $37-39^{\circ} \mathrm{C}$.

The trachea was intubated with a $6.0 \mathrm{~mm}$ standard cuffed endotracheal tube. For venous and arterial access / pressure monitoring, respectively, $8 \mathrm{Fr}$ umbilical catheters were inserted into the internal jugular vein and carotid artery through incisions in the neck as previously described [6]. Vital parameters including arterial and central venous blood pressure, ECG, rectal temperature and arterial blood chemistry (ABG; Stat profile ${ }^{\circledR}$, Nova Biomedical, Waltham, MA) were monitored during a stabilization period of $30 \mathrm{~min}$ and throughout the duration of the protocol.
After completion of catheter placement and stabilization, maintenance anesthesia was initiated as follows: sufentanil infusion at $0.2-0.3 \mu \mathrm{g} / \mathrm{kg} / \mathrm{hr}$ and isoflurane as $1 \%$ in $100 \%$ oxygen with supplemental ketamine boluses of $10 \mathrm{mg} / \mathrm{kg}$ as needed. Pancuronium was then administered as a $0.2 \mathrm{mg} / \mathrm{kg}$ intravenous bolus hourly, or as indicated by spontaneous diaphragmatic activity. Depth of anesthesia was assessed using changes in vital signs as primary criteria as well as response to tail clamping, and when appropriate, the infusion rate of sufentanyl was titrated as needed.

After allowing 30 minutes for baseline stabilization, vital parameters were recorded and the following measurements were obtained: pulmonary mechanics (NICO, Respironics, Wallingford, CT), arterial blood gas, and blood samples for subsequent analysis of inflammatory mediators associated with acute lung injury.

\section{OLV Protocol}

Following baseline measurements, the left primary bronchus was blocked under direct vision using a fiberoptic bronchoscope. This was done using an airway adaptor such that ventilation was not interrupted. The piglet was turned to the right lateral position to simulate surgical positioning during OLV. After the animal was positioned, the lung was blocked by inflating the cuff of the bronchial blocker under direct vision using a fiberoptic scope. To simulate thoracoscopic instrumentation, the $5 \mathrm{~mm}$ trocar was then placed through the non-dependent thoracic wall between the 7th and 8 th rib following local anesthesia with $1 \%$ lidocaine. Measurement sets were repeated immediately following initiation of OLV for comparison to bilateral lung ventilation, and then every half hour throughout the experiment. Breath sounds were periodically checked by auscultation, and when necessary, position of the bronchial blocker was rechecked using the fiberoptic bronchoscope.

Following $3 \mathrm{hrs}$ of OLV, measurements were repeated and the bronchial blocker was retrieved after deflating the balloon to restore bilateral ventilation. Three deep breaths were given manually to recruit alveoli, the animal was turned back to the supine position, and ventilation was continued for another 30 minutes before a final set of measurements was performed for comparison to pre-OLV data. The anesthesia was then deepened by administration of $20 \mathrm{mg} / \mathrm{kg}$ ketamine and $2-4 \mu \mathrm{g} / \mathrm{kg}$ of sufentanyl intravenously. A sternotomy was performed and the lungs were perfused with Millonig's buffer to flush the blood from the vasculature. Lung tissue from both the right and left lungs were harvested and fixed in $10 \%$ buffered formalin for gross histological analysis.

\section{Inflammatory Mediator Analysis}

The levels of TNF $\alpha$, IL- 6 and IL-8 in plasma samples were measured with quantitative ELISA using porcine specific Quantikine ELISA kits (R \& D systems, Minneapolis, $\mathrm{MN})$. Serum was appropriately diluted to fall within the detection range of each assay, and all standards and samples were assayed in duplicate. The test sensitivity for respective immunoassays was as follows: TNF- $\alpha$ (3.7 pg/ml), IL-6 (10 $\mathrm{pg} / \mathrm{ml})$ and IL-8 $(0.039 \mathrm{pg} / \mathrm{ml})$. Inter-assay and intra-assay coefficients of variance are $<10 \%$. 


\section{Histological Sample Preparation}

Fixed lung tissue from each animal was removed from formalin after 24 to $48 \mathrm{hr}$, embedded in paraffin and sectioned for slide preparation. Slides from each tissue section were stained with hematoxylin and eosin, and random fields from each section were digitally imaged for qualitative analysis.

\section{RESULTS}

Seven animals were used for the validation of this model. Piglets were $21 \pm 1$ days old and weighed $7.7 \pm 0.5 \mathrm{~kg}$.

\section{Physiologic Stability}

All animals demonstrated physiology typical of the clinical scenario (Table 1). All animals survived the protocol without the need for resuscitative pharmacologic support. Cardiovascular parameters as well as arterial $\mathrm{CO}_{2}$ tension and $\mathrm{pH}$ were stable throughout, independent of ventilation strategy. Arterial oxygen tension dropped to $62 \%$ of baseline with the onset of OLV and returned to $85 \%$ of baseline when BLV was restored.

Table 1. Physiologic Parameters During the Protocol

\begin{tabular}{|c|c|c|c|c|c|}
\hline & BLV & \multicolumn{3}{|c|}{ OLV } & BLV \\
\cline { 2 - 6 } & Baseline & $\mathbf{0 ~ h r}$ & $\mathbf{1 . 5}$ hr & $\mathbf{3 ~ h r}$ & End \\
\hline \hline $\begin{array}{c}\text { Rectal Temp } \\
\left({ }^{\circ} \mathrm{C}\right)\end{array}$ & $36.6 \pm 0.3$ & $37.4 \pm 0.2$ & $38.1 \pm 0.1$ & $38.8 \pm 0.1$ & $38.2 \pm 0.3$ \\
\hline $\begin{array}{c}\text { Mean } \\
\text { Arterial } \\
\text { Pressure } \\
(\mathrm{kPa})\end{array}$ & $9.7 \pm 0.2$ & $10.5 \pm 0.4$ & $10.3 \pm 0.2$ & $9.7 \pm 0.3$ & $9.1 \pm 0.6$ \\
\hline $\begin{array}{c}\mathrm{Heart} \\
\mathrm{Rate}\end{array}$ & $108 \pm 7$ & $108 \pm 8$ & $109 \pm 7$ & $112 \pm 9$ & $117 \pm 10$ \\
\hline $\begin{array}{c}\mathrm{Ph} \\
\mathrm{Ph}\end{array}$ & $7.40 \pm 0.09$ & $7.33 \pm 0.04$ & $7.34 \pm 0.03$ & $7.39 \pm 0.01$ & $7.46 \pm 0.02$ \\
\hline $\begin{array}{c}\text { PaCO } \\
(\mathrm{mmHg})\end{array}$ & $4.2 \pm 0.1$ & $5.3 \pm 0.1$ & $4.7 \pm 0.1$ & $5.0 \pm 0.2$ & $4.7 \pm 0.1$ \\
\hline $\begin{array}{c}\text { PaO } \\
(\mathrm{mmHg})\end{array}$ & $67.7 \pm 3.4$ & $41.9 \pm 3.9$ & $50.5 \pm 4.3$ & $57 \pm 3.5$ & $57.7 \pm 2.9$ \\
\hline
\end{tabular}

Data are expressed as mean \pm SEM; $n=7$ animals.

\section{Pulmonary Mechanics}

Table 2 shows the trends in pulmonary compliance and resistance over the course of the study. With the onset of OLV, respiratory compliance dropped to $88 \%$ of baseline and resistance increased to $148 \%$ of baseline. The trend values held steady throughout the duration of OLV and were restored to baseline values when BLV was restored at the end of the protocol.

Table 2. Pulmonary Mechanics During the Protocol

\begin{tabular}{|c|c|c|c|c|c|}
\hline & BLV & \multicolumn{3}{|c|}{ OLV } & BLV \\
\cline { 2 - 6 } & Baseline & $\mathbf{0 ~ h r}$ & $\mathbf{1 . 5} \mathbf{~ h r}$ & $\mathbf{3 ~ h r}$ & End \\
\hline \hline $\begin{array}{c}\text { Compliance } \\
\left(\mathrm{ml} / \mathrm{cmH}_{2} \mathrm{O}\right)\end{array}$ & $9.5 \pm 0.8$ & $8.0 \pm 0.6$ & $7.8 \pm 0.4$ & $7.1 \pm 0.3$ & $8.6 \pm 0.6$ \\
\hline $\begin{array}{c}\text { Resistance } \\
\left(\mathrm{cmH} \mathrm{H}_{2} \mathrm{O} / \mathrm{L} / \mathrm{min}\right)\end{array}$ & $12.5 \pm 0.6$ & $18.5 \pm 1.0$ & $17.5 \pm 0.3$ & $18.6 \pm 0.6$ & $14.8 \pm 1.4$ \\
\hline
\end{tabular}

Data are expressed as mean \pm SEM; $\mathrm{n}=7$ animals.

\section{Inflammatory Markers}

Fig. (2) shows the plasma TNF- $\alpha$ and IL-6 levels over the time course of the protocol for the seven piglets. Both TNF- $\alpha$ and IL- 6 increased markedly by the end of the experiment.

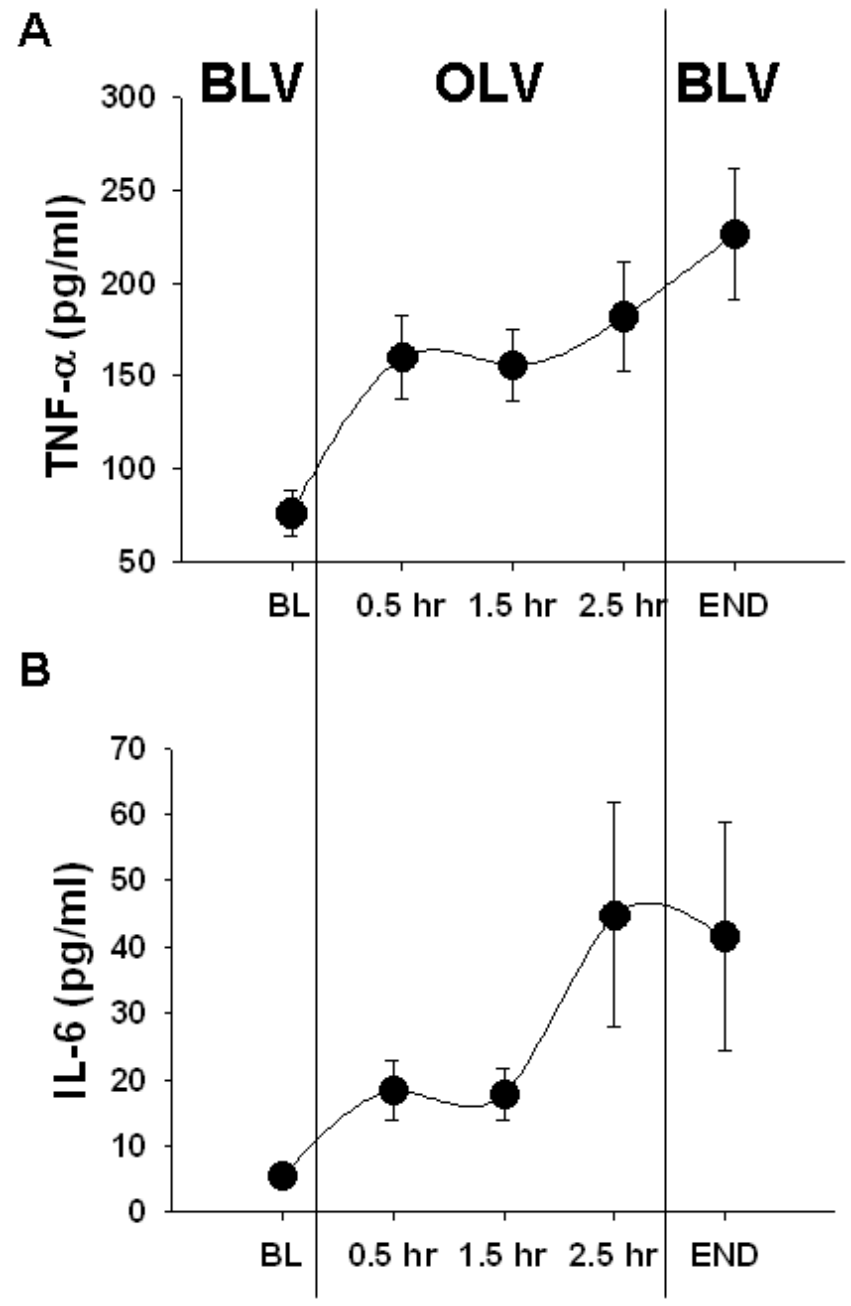

Fig. (2). Inflammatory mediator profiles for the seven piglets during the one lung ventilation protocol. (A) TNF- $\alpha$ increased sharply from baseline (BL) bilateral ventilation through the $0.5,1.5$ and 2.5 $\mathrm{hr}$ time points of OLV, and following the restoration of bilateral ventilation (END). The increase in TNF- $\alpha$ by end of the experiment was $197 \%$ when compared to baseline values. (B) Similarly, IL-6 rose throughout the duration of the experiment and had increased by $651 \%$ by end of the experiment.

\section{DISCUSSION}

Based on these findings, the juvenile piglet model is valid to represent the scenario of OLV during pediatric thoracoscopic surgeries. The piglet model was selected because of its comparable size to the human pediatric patient, and its previous application to the study of pediatric lung injury [6, 8]. A few studies have used larger pigs (25 to $35 \mathrm{~kg}$ ) for the study of lung functional parameters with OLV ventilation strategies [9, 10] or to study anesthetic agents [11, 12]. However, concerns can be raised, related to pulmonary anatomy and mechanics, for the application of this model as it relates to human patients. This report addresses and qualifies these concerns to justify the use of the piglet model for the study of OLV outcomes and potential therapies. 


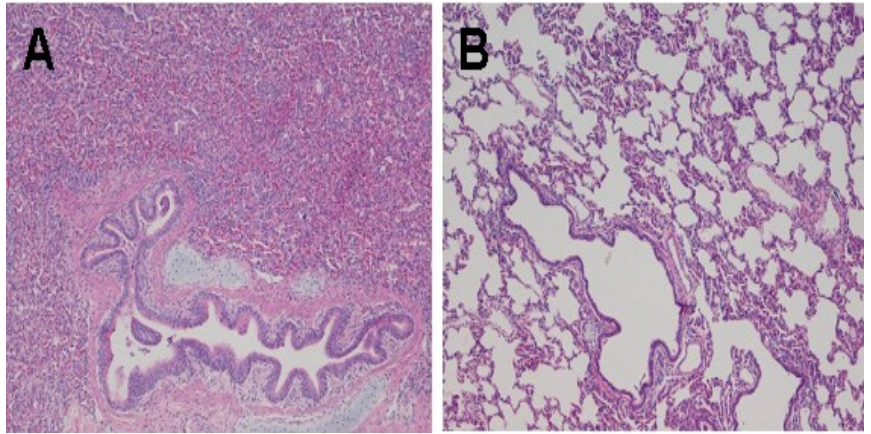

Fig. (3). (A) Represents the apical, anterior lung section from the left/collapsed lung and (B) represents the apical, anterior lung section from the right/ventilated lung of one piglet. Collapsed lung sections demonstrated greater degrees of atelectasis following 30 min of re-inflation and ventilation prior to harvest. Sections were imaged at 10x magnification.

Oral intubation of numerous animal species is known to be technically challenging. There is no significant obstacle to oral intubation of the juvenile piglets, which can be accomplished in a timely fashion using a conventional laryngoscope. Bronchial blockage requires the use of a fiberoptic bronchoscope to visualize the left main stem bronchus and to ascertain the effectiveness of the block itself. The timeline involved in this procedure is approximately 5-10 $\mathrm{min}$. The left primary bronchus was blocked, collapsing the left lung, because the swine right lung has an additional primary bronchus (apical bronchus) feeding the apical lobe; thus, complete blockage of the right lung could not be achieved practically.

Another significant anatomical difference between the pig and human is the shape of the chest wall, which impacts the pulmonary mechanics findings when transitioning between single and double lung ventilation. As compared to the pediatric human, the pig chest is more cathedral shaped with a proportionately greater anterior-posterior diameter. This relationship between anterior-posterior to lateral diameters gives the piglet a more stable chest wall compared to the human, with less tendency to collapse inward [13]. More significantly, the swine chest wall configuration allows for the lung to expand around the mediastinum. This chest configuration impacts the degree of chest wall displacement during OLV because the ventilated lung can expand into the contralateral thoracic space. In this regard, the impact on pulmonary compliance is different than what can be derived from literature for humans. Compliance in adult humans decreases between 40 and $60 \%$ with the transition to OLV $[4,14]$, compared to the $12 \%$ decrease reported here.

By convention, respiratory compliance accounts for the chest wall as well as the lung, unless intrathoracic pressure is measured, typically by esophageal manometry, and used in the derivation of compliance for the lung alone [7]. In the human patient, it is presumed that the chest wall compliance is unaffected by the transition to OLV, and the removal of $50 \%$ of the lung volume accounts for the approximately $50 \%$ decrease in respiratory compliance. In the piglet model, the more cathedral configuration of the chest wall allows the ventilated lung to expand around the pericardium, virtually eliminating chest wall compliance from the respiratory compliance equation when transitioned to OLV. Thus, the ap- proximately $50 \%$ reduction in lung volume is simultaneously counterbalanced by a removal of chest wall compliance, resulting in only a mild reduction in respiratory compliance values.

Despite the difference in respiratory mechanics compared to the human, the piglet model demonstrated similar outcomes in all other parameters reported for humans undergoing OLV. The hemodynamic and gas exchange parameters were indicative of the 15 to $40 \%$ shunt created by unilateral lung deflation [15]. When treated with the current standard for clinical care, the plasma inflammatory mediators increased in these animals in a manner similar to what is reported from clinical studies [5]. Although the clinical studies were unable to assess lung histomorphology, the piglets used to develop this model demonstrated clear histological differences between the two lungs, which serve as a basis for testing lung protective therapies.

\section{CONCLUSIONS}

These preliminary findings and the discussion presented here serve to validate the piglet model for studies of OLV by identifying similarities in clinical and physiological responses to OLV, while qualifying the disparities in anatomy and pulmonary mechanics between the human and pig models. We conclude that the piglet model can have great usefulness in the study of lung injury created by OLV and the efficacy of therapies aimed at attenuating this lung injury before clinical trials are mandated.

\section{ACKNOWLEDGEMENTS}

This study was funded through Nemours and NIH COBRE grant 1 P20 RR20173-01 (The Center for Pediatric Research).

\section{REFERENCES}

[1] Lammers CR, Hammer GB, Brodsky JB, et al. Failure to separate and isolate the lungs with an endotracheal tube positioned in the bronchus. Anesth Analg 1997; 85:946-7.

[2] Hammer GB, Brodsky JB, Redpath JH, et al. The univent tube for single-lung ventilation in paediatric patients. Paediatr Anaesth 1998; 8: 55-57.

[3] Hammer GB, Manos SJ, Smith BM, et al. Single-lung ventilation in pediatric patients. Anesthesiology 1996; 84: 1503-6.

[4] Tusman G, Bohm SH, Sipmann FS, et al. Lung recruitment improves the efficiency of ventilation and gas exchange during onelung ventilation anesthesia. Anesth Analg 2004; 98: 1604-9.

[5] Michelet P, D'Journo XB, Roch A, et al. Protective ventilation influences systemic inflammation after esophagectomy: A randomized controlled study. Anesthesiology 2006; 105: 911-9.

[6] Miller TL, Blackson TJ, Shaffer TH, et al. Tracheal gas insufflation-augmented continuous positive airway pressure in a spontaneously breathing model of neonatal respiratory distress. Pediatr Pulmonol 2004; 38: 386-95.

[7] Miller TL, Palmer C, Shaffer TH, et al. Neonatal chest wall suspension splint: A novel and noninvasive method for support of lung volume. Pediatr Pulmonol 2005; 39: 512-20.

[8] Miller TL, Singhaus CJ, Sherman TI, Greenspan JS, Shaffer TH. Physiologic implications of helium as a carrier gas for inhaled nitric oxide in a neonatal model of bethanecol-induced bronchoconstriction. Pediatr Crit Care Med 2006; 7: 159-64.

[9] Michelet P, Roch A, Brousse D, et al. Effects of peep on oxygenation and respiratory mechanics during one-lung ventilation. $\mathrm{Br} \mathrm{J}$ Anaesth 2005; 95: 267-73.

[10] McMullen MC, Girling LG, Graham MR, et al. Biologically variable ventilation improves oxygenation and respiratory mechanics during one-lung ventilation. Anesthesiology 2006; 105(1): 91-7.

[11] Schwarzkopf K, Schreiber T, Gaser E, et al. The effects of xenon or nitrous oxide supplementation on systemic oxygenation and 
pulmonary perfusion during one-lung ventilation in pigs. Anesth Analg 2005; 100: 335-9.

[12] Schwarzkopf K, Schreiber T, Bauer R, et al. The effects of increasing concentrations of isoflurane and desflurane on pulmonary perfusion and systemic oxygenation during one-lung ventilation in pigs. Anesth Analg 2001; 93: 1434-8.

[13] Palmer C, Roberts RL, Moffitt S, et al. Chest measurements reveal rib cage flattening in newborns with respiratory distress. Pediatr Res 2001; 49: 408A.
[14]

Schilling T, Kozian A, Huth C, et al. The pulmonary immune effects of mechanical ventilation in patients undergoing thoracic surgery. Anesth Analg 2005; 101: 957-65.

[15] Torda TA, McCulloch CH, O'Brien HD, et al. Pulmonary venous admixture during one-lung anaesthesia. The effect of inhaled oxygen tension and respiration rate. Anaesthesia 1974; 29: 272-9.

Received: August 31, 2008

Revised: September 6, 2008

Accepted: November 23, 2008

(C) Miller et al.; Licensee Bentham Open.

This is an open access article licensed under the terms of the Creative Commons Attribution Non-Commercial License (http://creativecommons.org/licenses/by$\mathrm{nc} / 3.0 /$ ) which permits unrestricted, non-commercial use, distribution and reproduction in any medium, provided the work is properly cited. 